\title{
Detection of Atrial Fibrillation on Stroke Units: Look Harder, Look Longer, Look in More Sophisticated Ways
}

\author{
Ying X. Gue ${ }^{a}$ Tatjana S. Potpara ${ }^{b}$ Gregory Y.H. Lip ${ }^{a, c}$ \\ aLiverpool Centre for Cardiovascular Science, University of Liverpool and Liverpool Heart \& Chest Hospital, \\ Liverpool, UK; ${ }^{b}$ Clinical Centre of Serbia \& School of Medicine, Belgrade University, Belgrade, Serbia; ${ }^{c}$ Aalborg \\ Thrombosis Research Unit, Department of Clinical Medicine, Aalborg University, Aalborg, Denmark
}

Atrial fibrillation (AF) is the commonest sustained cardiac arrhythmia, often asymptomatic and sometimes presenting for the first time with a serious complication related to AF, notably stroke or heart failure. Patients with AF commonly have other cardiovascular risk factors and non-cardiovascular conditions. In patients without known AF, an important message is to "think of AF ...," especially in those aged $\geq 65$ years or presenting for a clinical check-up for their comorbidity, before a complication occurs. What about patients without known AF who present with a stroke? What should we do about detecting $\mathrm{AF}$ and addressing comorbidities?

In those with known AF, the 2020 European Society of Cardiology guidelines for diagnosis and management of $\mathrm{AF}$ [1] recommend a structured characterization of AF using the 4S-AF scheme (Stroke risk; Symptom severity; Severity of AF burden; Substrate severity) [2] to better guide treatment decisions on thromboprophylaxis and rate versus rhythm management strategy. Apart from being an easy-to-remember guide for clinicians in managing $\mathrm{AF}$, the $4 \mathrm{~S}-\mathrm{AF}$ scheme also highlights the importance of recognizing the complexity of AF pathophysiology, including clinical characteristics, other comorbidities, and atrial structure and function which should be taken into consideration when evaluating a patient with new onset AF [3]. Management of AF focuses on a holistic approach using the simple ABC pathway ("A" anticoagulation/ avoid stroke; "B" better symptom management; "C" cardiovascular and comorbidity optimization) which has been associated with better clinical outcomes in multiple studies [4-6].

In those without known $\mathrm{AF}$, timely detection and diagnosis of incident AF is important to prevent the associated risks of stroke and mortality. There are many clinical risk factors related with the development of AF [7] and they are important to identify high risk patients who may benefit from screening for AF $[1,8]$. Currently, there are many available methods for screening for AF ranging from clinical examination to mobile health technologies [1]. There are, however, risks involved in mass screening, in particular misinterpretation leading to overdiagnosis and overtreatment which can be associated with harm [9]. Another challenge is the identification of atrial high-rate episodes (AHRE) or subclinical AF, as it remains unclear whether patients with AHRE would have the same net clinical benefit from oral anticoagulants as those with clinically overt AF. It is currently recommended [1] that in patients with AHRE, continued patient follow-up and

$\begin{aligned} & \text { karger@karger.com } \\ & \text { www.karger.com/ced }\end{aligned}$
Karger ${ }^{\prime /}$

Gregory Y.H. Lip

University of Liverpool, William Henry Duncan Building

6 West Derby Street

Liverpool L7 8TX (UK)

Gregory.Lip@liverpool.ac.uk 
Fig. 1. Detection of AF in post-stroke patients without known AF. AF, atrial fibrillation.

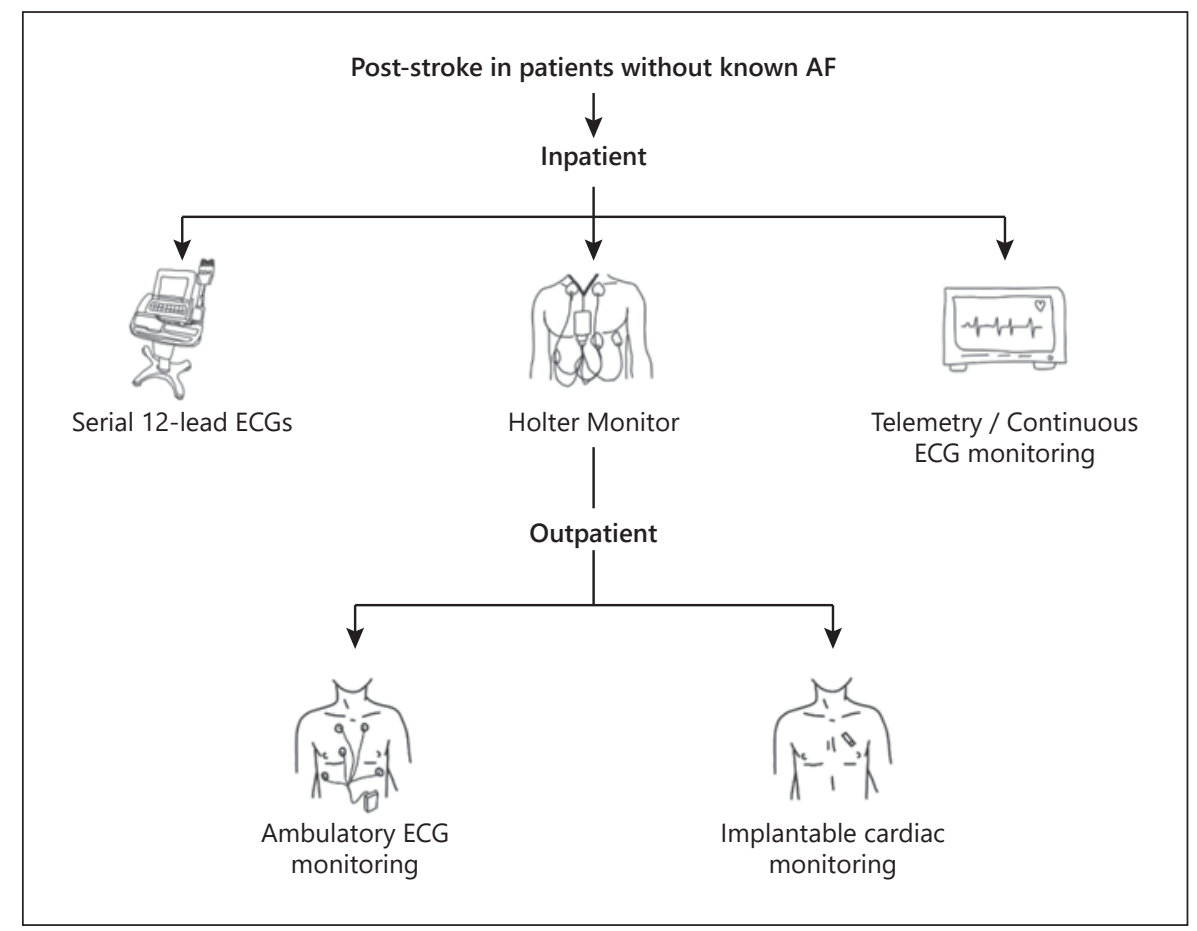

monitoring is required to detect progression to clinical $\mathrm{AF}$ and the use of OAC should be considered in patients with long-lasting AHRE and high/very high risk of stroke in whom a positive clinical benefit from OAC is anticipated.

The search for AF should not stop after the occurrence of an acute ischemic stroke. In fact, cardio-embolism associated with AF accounts for up to a third of all ischemic strokes [10]. AF, especially if paroxysmal, can be asymptomatic and, therefore, difficult to detect but asymptomatic $\mathrm{AF}$ carries the risk of stroke and mortality comparable to that with symptomatic AF [11]. Detection of previously unknown AF after acute ischemic stroke has important implications on the decision on future thromboprophylaxis treatment. In post-stroke patients, especially with embolic stroke of undetermined source, AF may play a larger role [12] thereby justifying a more prolonged and intensive search for AF [13].

With several RCTs establishing the efficacy of ECG monitoring in post-stroke AF detection [14, 15], the 2020 European Society of Cardiology AF guidelines [1] recommended short-term ECG recording followed by at least 72-h of continuous ECG monitoring post stroke. Up to $5.1 \%$ of post-stroke patients have AF detected as in-patients using serial ECGs, continuous ECG monitoring or telemetry [15]. A tiered longer ECG monitoring approach, identifying patients with higher risk clinical characteristics [7] to select for longer monitoring, including the insertion of an intracardiac monitor in case of cryptogenic stroke could be considered in order to increase detection rate, which could be up to $23.7 \%$ [15].

The detection of AF during the inpatient post stroke period primarily relies on clinicians reviewing ECGs and monitors manually. AF episodes may also occur remote to the episode of stroke or thromboembolism. With advances in machine learning and automation, the idea of simplifying the process of detecting AF using computerbased analysis with minimal physician input is no longer implausible [16]. Apart from identifying AF, more sophisticated methods can be used to identify other atrial abnormalities associated with AF such as premature atrial complexes and atrial tachycardia which can be used to identify patients at higher risk of AF. However, there are implications in fully relying on automation, including the risk involved with a false-positive diagnosis (leading to overtreatment which can be associated with harm) and a false negative (missing the diagnosis and potentially not initiating appropriate treatment).

In this issue of the journal, Rogalewski et al. [17] set out to compare the differences between manual and automated analysis of continuous ECG monitoring to detect $\mathrm{AF}$ in post-stroke patients without prior history of AF. In a large German stroke unit, a total of 216 consecutive patients with ischemic strokes were enrolled. In 555 analysis days compared, manual analysis detected $37 \mathrm{AF}$, 
whereas 57 were detected on automated analysis. Costs of analysis were also higher at USD 7.80 per ECG with automated and USD 2.10 per ECG for manual, calculated using per-hour cost for personnel. They concluded that although automatic AF detection is highly specific (94.6\%), its sensitivity is relatively low (78.4\%) when compared to the gold standard manual analysis and therefore should be complementary to manual ECG analysis rather than replacing it.

Although advancements in technology have the potential to reduce a proportion of manual work in the clinical setting, currently, there are still limitations as highlighted in the study. Therefore, while the promise of a fully automated system appears appealing, clinicians should approach that with a level of healthy skepticism. As a general rule, $\mathrm{AF}$ is more likely to be detected if we look longer, look harder and if we look using more sophisticated ways (Fig. 1).

\section{Conflict of Interest Statement}

The authors have no conflicts of interests to disclose.

\section{Funding Sources}

This article was not funded by any external sources.

\section{Author Contributions}

Y.X.G.: conceptualization and writing - original draft. T.P.: conceptualization and writing - review and editing. G.Y.H.L.: conceptualization and writing - review and editing, corresponding author.

\section{References}

1 Hindricks G, Potpara T, Dagres N, Arbelo E, Bax JJ, Blomström-Lundqvist C, et al. 2020 ESC guidelines for the diagnosis and management of atrial fibrillation developed in collaboration with the european association of cardio-thoracic surgery (EACTS). Eur Heart J. 2020 Aug.

2 Potpara TS, Lip GYH, Blomstrom-Lundqvist C, Boriani G, Van Gelder IC, Heidbuchel H, et al. The 4S-AF scheme (stroke risk; symptoms; severity of burden; substrate): a novel approach to in-depth characterization (rather than classification) of atrial fibrillation. Thromb Haemost. 2020 Aug.

3 Staerk L, Sherer JA, Ko D, Benjamin EJ, Helm RH. Atrial fibrillation: epidemiology, pathophysiology, clinical outcomes. Circ Res. 2017 Apr;120(9):1501-17.

4 Guo Y, Lane DA, Wang L, Zhang H, Wang H, Zhang W, et al. Mobile health technology to improve care for patients with atrial fibrillation. J Am Coll Cardiol. 2020 Apr;75(13): 1523-34.

5 Yoon M, Yang PS, Jang E, Yu HT, Kim TH, Uhm JS, et al. Improved population-based clinical outcomes of patients with atrial fibrillation by compliance with the simple $\mathrm{ABC}$ (Atrial Fibrillation Better Care) pathway for integrated care management: a nationwide cohort study. Thromb Haemost. 2019 Oct; 119(10):1695-703.

6 Proietti M, Romiti GF, Olshansky B, Lane DA, Lip GYH. Comprehensive management with the $\mathrm{ABC}$ (atrial fibrillation better care) pathway in clinically complex patients with atrial fibrillation: a post hoc ancillary analysis from the AFFIRM trial. J Am Heart Assoc. 2020 May.

7 Aronson D, Shalev V, Katz R, Chodick G, Mutlak D. Risk score for prediction of 10-year atrial fibrillation: a community-based study. Thromb Haemost. 2018;118(9):1556-63.

8 Guo Y, Wang H, Zhang H, Chen Y, Lip GYH. Population-based screening or targeted screening based on initial clinical risk assessment for atrial fibrillation: a report from the huawei heart study. J Clin Med. 2020 May; 9(5):1493.

9 Mairesse GH, Moran P, Van Gelder IC, Elsner C, Rosenqvist M, Mant J, et al. Screening for atrial fibrillation: a european heart rhythm association (EHRA) consensus document endorsed by the heart rhythm society (HRS), asia pacific heart rhythm society (APHRS), and sociedad latinoamericana de estimulación cardíaca y electrofisiolog. Europace. 2017 Oct;19(10):1589-623.

10 Friberg L, Rosenqvist $M$, Lindgren A, Terént A, Norrving B, Asplund K. High prevalence of atrial fibrillation among patients with ischemic stroke. Stroke. 2014 Sep;45(9):2599-605.

11 Boriani G, Laroche C, Diemberger I, Fantecchi E, Popescu MI, Rasmussen LH, et al. Asymptomatic atrial fibrillation: clinical correlates, management, and outcomes in the EORP-AF pilot general registry. Am J Med. 2015 May;128(5):509-e2.
12 Wachter R, Freedman B. The role of atrial fibrillation in patients with an embolic stroke of unknown source (ESUS). Thromb Haemost. 2017;117(10):1833-5.

13 Israel C, Kitsiou A, Kalyani M, Deelawar S, Ejangue LE, Rogalewski A, et al. Detection of atrial fibrillation in patients with embolic stroke of undetermined source by prolonged monitoring with implantable loop recorders. Thromb Haemost. 2017 Nov; 117(10):1962-9.

14 Kishore A, Vail A, Majid A, Dawson J, Lees KR, Tyrrell PJ, et al. Detection of atrial fibrillation after ischemic stroke or transient ischemic attack: a systematic review and metaanalysis. Stroke. 2014 Feb;45(2):520-6.

15 Sposato LA, Cipriano LE, Saposnik G, Ruíz Vargas E, Riccio PM, Hachinski V. Diagnosis of atrial fibrillation after stroke and transient ischaemic attack: a systematic review and meta-analysis. Lancet Neurol. 2015 Apr;14(4): 377-87.

16 Adami A, Gentile C, Hepp T, Molon G, Gigli GL, Valente M, et al. Electrocardiographic RR interval dynamic analysis to identify acute stroke patients at high risk for atrial fibrillation episodes during stroke unit admission. Transl Stroke Res. 2019 Jun;10(3):273-8.

17 Rogalewski A, Plumer J, Feldmann T, Oelschlager C, Greeve I, Kitsiou A, et al. Detection of atrial fibrillation on stroke units: comparison of manual versus automatic analysis of continuous telemetry. Cerebrovasc Dis. 2020 . 